\title{
Influencia del gobierno corporativo en las empresas del Ecuador, y su aporte a la competitividad internacional
}

Fecha de recepción: 03-12-2020 • Fecha de aceptación: 20-01-2021 • Fecha de publicación: 10-02-2021

Fernando Gustavo Pérez Sisa

Universidad Central del Ecuador

fjperez@uce.edu.ec

https://orcid.org/0000-0002-6279-4366

\section{RESUMEN:}

El gobierno corporativo es un tema amplio e interesante, la investigación académica ha entregado varios hallazgos importantes, mismos que han sido acogidos por varias corporaciones a nivel mundial, de manera que en el Ecuador existen empresas grandes que podrían adoptar estas prácticas para ser competitivos internacionalmente. En el presente estudio se analiza la influencia del gobierno corporativo en las grandes empresas del Ecuador y su aporte en la competitividad internacional, tomando en cuenta los diferentes elementos que están cambiando el mundo globalizado, determinando así, el impacto que este posee dentro de la captación de inversiones, fusiones corporativas, flujos de información y procesos jerárquicos, permitiendo alcanzar una eficiencia y posicionamiento en mercados mundiales. Mediante la aplicación de encuestas y un modelo teórico documental se determinó la influencia de este en el desarrollo nacional e internacional de las organizaciones ecuatorianas, mismas que al manejar buenas prácticas empresariales tienden a ser más competitivas en el mercado. 


\section{ABSTRACT}

Corporate governance is a broad and interesting topic, academic research has delivered several important findings, which have been embraced by several corporations worldwide, so that in Ecuador there are large companies that could adopt these practices to be competitive internationally. Analyzing the influence of corporate governance on large companies in Ecuador and its contribution to international competitiveness, taking into account the different elements that are changing the globalized world, it is possible to determine the impact that it has within the attraction of investments, corporate mergers, information flows and hierarchical processes, allowing to achieve efficiency and positioning in world markets. Through the application of surveys and a theoretical documentary model, the influence of this on the national and international development of Ecuadorian organizations was determined. These organizations, when handling good business practices, tend to be more competitive in the market. 


\section{Introducción}

Las grandes empresas, tanto públicas como privadas, han buscado un modelo económico que logre llevarlas al desarrollo y crecimiento mediante estrategias de importación y exportación, estas pueden insertarse en el mercado global.

El gobierno corporativo posee un impacto relevante a nivel internacional, varios autores consideran que un adecuado gobierno corporativo en las organizaciones permite manejar lo diversos retos que trae la globalización (Gómez, 2018, pág. 3).

Dentro del gobierno corporativo se encuentra un conjunto de normas, procedimientos y principios que apoyan a una eficiencia en la estructura organizacional y un funcionamiento óptimo en las empresas, esto se debe a las interrelaciones entre los accionistas, la junta directiva, el consejo administrativo, y cada una de las áreas que conforman la organización. Este plantea reglas y herramientas para optimizar la toma de decisiones y generar valor competitivo.

A s vez, con el pasar de los años, ha generado una ventaja competitiva, siendo así que en el año 2004 la Organización para la Cooperación y Desarrollo Económico (OCDE) se convirtió en una de las entidades internacionales encargadas de la difusión e instrucción de las buenas prácticas del gobierno corporativo entre sus países miembros.

Esta entidad se encuentra conformada por dos comités, el primero se encarga de guardar y velar por el cumplimiento de cada uno de los principios del gobierno corporativo, mientras que al segundo se le atribuyen funciones orientadas hacia las directrices y mecanismos de gobierno corporativo que las empresas públicas y privadas pueden aplicar para obtener una ventaja competitiva.

El determinar su influencia en las grandes empresas del Ecuador representa un factor clave para la competitividad, mediante una revisión teórico documental que plantea recolectar datos y herramientas que faciliten el desarrollo de una propuesta que permita a las organizaciones contar con una mayor presencia en el mercado nacional e internacional.

Uno de los problemas que suele suscitarse en el gobierno corporativo se debe a las partes interesadas tanto internas (propietarios, ejecutivos, personal) como externas (proveedores, clientes, autoridades y sociedad en general) a la organización, por lo expresado anteriormente es prioritario desarrollar un sistema que integre de forma equitativa a cada una de las partes, en base a lineamientos articulados al código de gobierno corporativo andino y políticas internacionales.

Por consiguiente, las grandes empresas ecuatorianas tienen poca presencia internacional, ocasionando dificultad para competir en el mercado globalizado sin caracterizar que el gobierno corporativo, en las organizaciones del Ecuador, aporta a la competitividad debido a que brinda herramientas claras para optimizar su sistema, obtener ventaja competitiva y desarrollarse.

Las grandes empresas públicas y privadas del país, para lograr presencia en el mercado nacional e internacional, deben aumentar su nivel de productividad, para ello deben incrementar su 
capacidad de sostener e incrementar la participación en el mercado (Porter, 1990, pág. 2).

En la Tabla 1 se visualizará de manera clara como el Ecuador ha ido evolucionando su posición en los índices de competitividad global.

Tabla 1.

Ranking de Competitividad

\begin{tabular}{|l|l|l|}
\hline Año & Ranking de competitividad (puesto) & Índice de competitividad \\
\hline 2019 & $90^{\circ}$ & 55,74 \\
\hline 2018 & $86^{\circ}$ & 55,85 \\
\hline 2017 & $97^{\circ}$ & 55,86 \\
\hline 2016 & $76^{\circ}$ & 58,18 \\
\hline 2014 & $71^{\circ}$ & 59,69 \\
\hline 2013 & $86^{\circ}$ & 56,31 \\
\hline 2012 & $101^{\circ}$ & 54,61 \\
\hline 2011 & $105^{\circ}$ & 52,17 \\
\hline 2010 & $105^{\circ}$ & 50,81 \\
\hline
\end{tabular}

Fuente: adaptación de Revista Expansión (2019).

Como se evidencia en la tabla anterior, en el 2019 Ecuador obtuvo 55,74 puntos en el índice de competitividad, publicado por el Foro Económico Mundial (FEM), mismo que se encarga de medir cómo utiliza un país sus recursos y capacidad para proveer a sus habitantes de un alto nivel de prosperidad. Cabe mencionar que este ha disminuido la puntuación respecto al informe de 2018, en el que en el que obtuvo 55,85 puntos. Ese valor sitúa a Ecuador en el puesto número 90, es decir, que tiene un nivel de competitividad mundial bastante deficiente si lo comparamos con el resto de los 141 países del ranking, pero ha empeorado su situación en relación a datos aportados por el año 2018, mismo que ocupaba el puesto 86 (Expansión, 2019).

La presente investigación analizará como el gobierno corporativo funciona dentro de las 10 principales empresas del Ecuador, su impacto y participación en el mercado internacional, buscando una guía de estudio que se ajuste al contexto económico actual.

\subsection{Buenas prácticas del gobierno corporativo}

El gobierno corporativo es una herramienta que facilita la promoción en las gestiones empresariales con un esquema de anticipación y manejo de riesgos, fomentando un ambiente de claridad y confianza para todos los interesados en el desarrollo de una empresa.

Contar con buenas prácticas aportan al fortalecimiento de la capacidad estratégica de las organizaciones. Es decir, al cuantificar los objetivos del negocio (ventas, rentabilidad) se puede mejorar la praxis operativa y generar continuidad en cada uno de los procesos (Cárdenas, 2018, pág. 1).

Un buen manejo de esta herramienta resulta de gran relevancia en las grandes y pequeñas 
empresas porque fortalece las capacidades estratégicas, el control y la administración eficiente de las organizaciones.

Por lo descrito anteriormente, es necesaria la implementación de buenas prácticas de gobierno corporativo en las organizaciones que faciliten la adopción de normas técnicas internacionalmente aceptadas para que garanticen el acceso a créditos internacionales.

La creación de un instituto o un ente regulador ecuatoriano facilitará su implementación en las empresas ecuatorianas, como también, este ente jugará un papel importante en las relaciones con los inversionistas, y su adaptación en un mercado globalizado.

\subsection{Modelo de transparencia y la inversión internacional}

En la actualidad existen acuerdos de inversión internacional en que se pide a las empresas transnacionales divulgar determinada información. En el Ecuador existe La Ley Orgánica de Transparencia y Acceso a la Información Pública (LOTAIP) que plantea la participación ciudadana y el derecho de acceso a la información relacionada con asuntos públicos, para ejercer un efectivo control y exigir la rendición de cuentas a las instituciones gubernamentales o aquellas que perciben recursos estatales (UNCTAD, 2004, pág. 1).

El Consejo de Participación Ciudadana y Control Social (CPCCS) del Ecuador propone a las entidades del sector público un modelo de prácticas transparentes que ayudaran a estructurar un sistema administrativo enfocado a la discreción de los diferentes servidores en la toma de decisiones y ejecución de sus funciones.

En relación a la inversión internacional, el crecimiento económico de un país no puede ser explicado sin hacer referencia a las diversas actividades de comercio internacional.

La economía ecuatoriana no registró en 2019 cifras favorables en diferentes rubros. Uno de ellos, la inversión extranjera directa, que continúa estancada pese a la aprobación de leyes que, según los voceros de Gobierno, mejoraría esta situación. Según cifras aportadas por el Banco Central de Ecuador (BCE), la inversión extranjera directa, entre los meses de enero y octubre de 2018, fue de USD \$851 millones. Mientras que, en el mismo periodo, en 2019 bajó a USD \$610 millones (Peralta, 2020).

Un modelo de transparencia adecuado permitirá generar mayor inversión internacional en el país, a través del conocimiento y la divulgación de datos claves de las organizaciones como la parte presupuestaria, legal, procedimientos, etc., que ayuden a recuperar la confianza de las empresas extranjeras (Bernal, 2019).

\subsection{La responsabilidad social en el gobierno corporativo}

El gobierno corporativo debe garantizar la alineación estratégica de la organización, el control y manejo efectivo de la alta dirección estratégica, consejo administrativo y la rendición de cuentas 
ante la empresa, los accionistas y el estado.

Cada consejo de estructura y procedimientos varía dependiendo del país en que se vaya a implementar, en algunas ocasiones consiste en atribuir la función de supervisión y gestión a dos órganos distintos. Generalmente estos sistemas poseen un consejo de supervisión, formado por varios miembros, tanto del consejo ejecutivo como administrativo (OCDE, 2016).

La responsabilidad social permite desarrollar una imagen positiva de las organizaciones en la sociedad nacional e internacional, actuando sobre las áreas sociales, laborales, micro ambiental y el respeto a los derechos humanos.

\subsection{Gobierno corporativo y la asociatividad empresarial}

La asociatividad empresarial emerge con la necesidad de innovación, crecimiento, desarrollo y frontera de oportunidades de las pequeñas y medianas empresas tienen frente a un mundo globalizado mismo en el que cada día resulta más difícil mantener su posicionamiento, competitividad y desarrollo sostenible (Sisa, 2020).

Dada la exigencia de los estándares de calidad y mercados mundiales, las empresas pequeñas y medianas no logran penetrar en los mismos, pues poseen una capacidad productiva pequeña dificultando satisfacer los niveles de demanda en los potenciales clientes. Es ahí, donde la actividad asociativa constituye una propuesta innovadora para las PYMES, debido a que facilitan un aporte competitivo frente a los grandes desafíos internacionales (Sisa, 2020).

De acuerdo a lo anterior, el gobierno corporativo va de la mano con los diferentes modelos de asociatividad empresarial, debido a que facilita la unión de organizaciones públicas o privadas de diferentes escalas, aportando con un sistema de cargos y responsabilidades conciso y flujos de información continua (Acción y Desarrollo, 2020).

\subsection{Intereses del gobierno corporativo}

La investigadora Shirley Peña manifiesta dentro de su investigación "Influencia del Gobierno Corporativo en las Empresas Familiares" que estas se ven afectadas por diversos intereses entre los cuales podemos destacar:

\section{- Estructura de Capital}

Dentro de las organizaciones las distintas ganancias operacionales se ven afectadas por las distintas decisiones generacionales (padres o hijos), mismos que son bajo fundamentos homogéneos generando problemas en su distribución y capacidad de reacción. Ante esos escenarios, el gobierno corporativo aporta a las organizaciones una estructura funcional y en base a cargos y montos de distribución para el capital mismo que se solidifica y plantea en base a las leyes y políticas de cada país (Peña, S., 2020). 
- La aversión al riesgo

La aversión o rechazo al riesgo genera diferentes problemas en los negocios donde predomina un sistema familiar, esto provoca una preferencia para las inversiones de capital, lo que genera un estancamiento en los sistemas de captación de capital en las organizaciones sin un gobierno corporativo establecido y centrado en un orden funcional (Peña, S., 2020).

- Política de dividendos

Es importante establecer una política de dividendos y repartición de ganancia entre los socios que esté fundamentada en los aportes y crecimiento sostenible de la organización, esto puede generar controversia en empresas familiares, donde no se encuentra clara los niveles de aportes o ingresos por empleados, dificultando o incluso llevando a las organizaciones a divisiones y conflictos internos, en base a lo anterior, el gobierno corporativo aporta con un sistema ajustado a las regulaciones gubernamentales, especificando los montos, niveles de jerarquía y división de utilidades (Bolsa de Comercio de Buenos Aires, 2020).

\subsection{Contabilidad y gobierno corporativo}

La integración financiera dentro de las organizaciones integra rasgos básicos de las políticas y normativas que manejen los diversos países, mismos que están regulados por los conceptos estandarizados de contabilidad, estos van de la mano con el gobierno corporativo, debido a que aportan con una visión más compleja de los procesos que conforman la economía organizacional (López, 2020).

\subsection{Estructura y composición del gobierno corporativo}

Dentro de las organizaciones, el gobierno corporativo es implementado mediante democracia, donde los miembros principalmente son: la junta o asamblea de accionistas, directorio ejecutivo y la planta de gerentes, dentro de la Tabla 2 y Figura 1 se lo aborda con más profundidad (Urriza, Gobernabilidad y Gobierno Corporativo, 2017). 
Tabla 2.

Estructura del Gobierno Corporativo

\begin{tabular}{|l|l|l|}
\hline $\begin{array}{l}\text { ORGANISMOS DEL } \\
\text { GOBIERNO CORPORARIVO }\end{array}$ & AGENTES INVOLUCRADOS & PRINCIPALES FUNCIONES \\
\hline $\begin{array}{l}\text { Junta o asamble de } \\
\text { Accionistas. }\end{array}$ & $\begin{array}{l}\text { Accionistas o Propietarios de la } \\
\text { organización. }\end{array}$ & $\begin{array}{l}\text { Aportan capital y toman } \\
\text { decisiones. }\end{array}$ \\
\hline Directorio o junta directiva. & Accionistas Direores. & $\begin{array}{l}\text { Aportan capital y toman } \\
\text { decisiones. }\end{array}$ \\
\hline Planta de gerentes. & $\begin{array}{l}\text { Gerente general y Gerentes de las } \\
\text { distintas areas de la organización. }\end{array}$ & $\begin{array}{l}\text { Toman decisiones estrategicas } \\
\text { en base a las decisiones del } \\
\text { directorio y la asamblea de } \\
\text { accionistas. }\end{array}$ \\
\hline
\end{tabular}

Fuente: Gobernabilidad y gobierno corporativo: análisis y medición de su eficacia en organizaciones (Urriza, Gobernabilidad y Gobierno Corporativo, 2017)

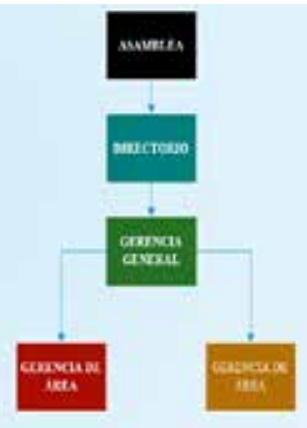

Figura 1. Estructura del Gobierno Corporativo

Fuente: Gobernabilidad y gobierno corporativo: análisis y medición de su eficacia en organizaciones (Urriza, Gobernabilidad y Gobierno Corporativo, 2017).

\section{Principios para la práctica de un buen gobierno corporativo}

En base a el modelo aportado por la Organización para la Cooperación y Desarrollo Económico en América Latina, los principios fundamentales para un óptimo manejo del gobierno corporativo se los plasma detalladamente en la Figura 2. 


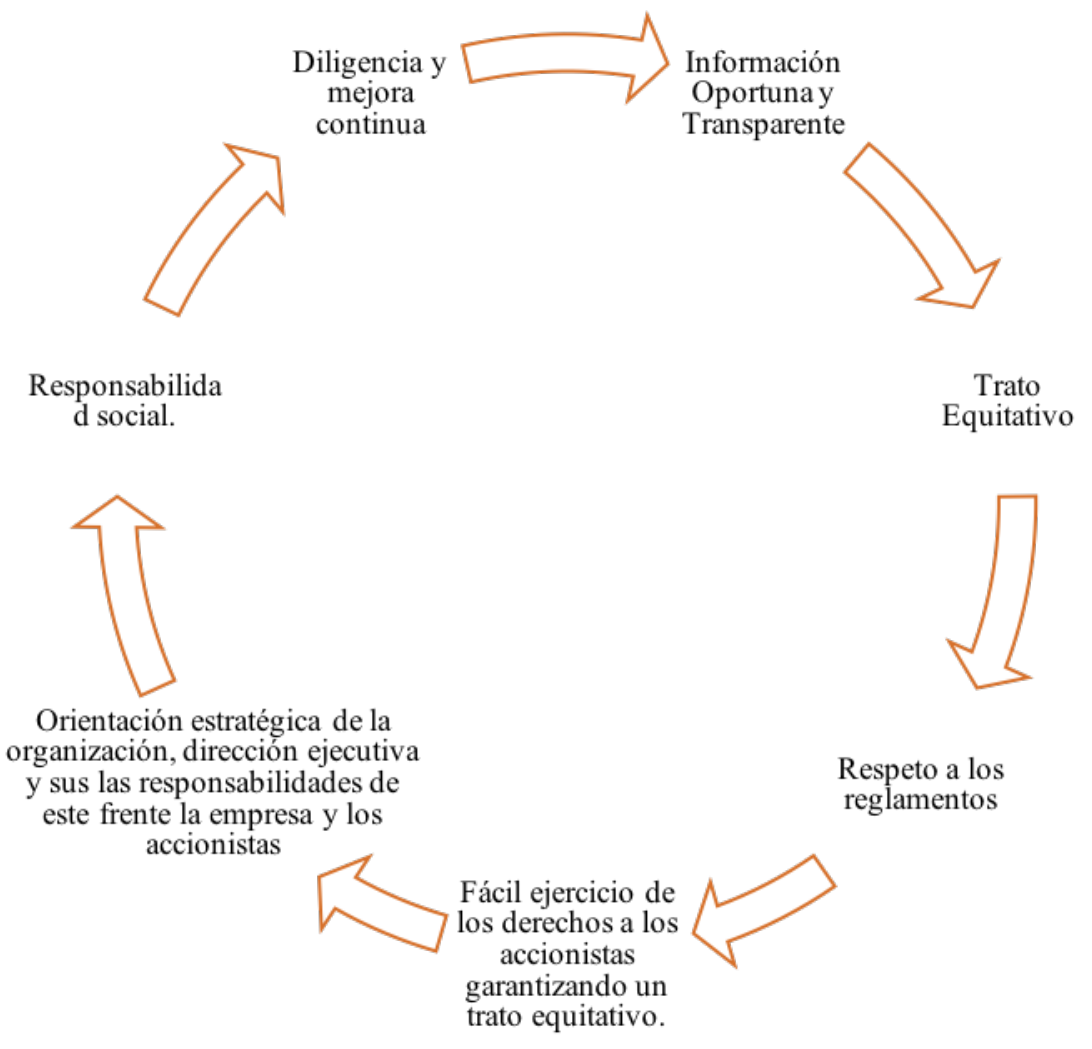

Figura 2. Principios del Gobierno Corporativo

Fuente: Gobernabilidad y gobierno corporativo: análisis y medición de su eficacia en organizaciones (Urriza,

Gobernabilidad y Gobierno Corporativo, 2017).

\subsection{Análisis y medición de la eficacia del gobierno corporativo en las organizaciones}

El adecuado manejo del gobierno corporativo se relaciona con el crecimiento empresarial y la supervivencia de la organización ante los diferentes escenarios externos o internos que se puedan presentar, garantizando la permanencia y continuidad de la organización a lo largo del tiempo (Urriza, Gobernabilidad y Gobierno Corporativo, 2017).

Para medir adecuadamente su impacto es necesario implementar indicadores de desempeño organizacional enfocándose en la realidad empresarial y asociatividad de cada una de sus áreas (Urriza, Gobernabilidad y Gobierno Corporativo, 2017).

Debido a la complejidad que se puede presentar para medir con exactitud los beneficios o eficacia del gobierno corporativo, es aconsejable considerar los siguientes criterios:

- Claridad: indicador que mide el flujo de información exacta e inequívoca de la organización.

- Relevancia: ajustar los indicadores en base a la realidad empresarial, entorno y diferentes variables que se puedan tomar en cuenta. 
- Económico: la medición empresarial debe estar sujeta a los presupuestos organizacionales.

- Medible: los diferentes criterios que se tomen en cuenta para la medición del gobierno corporativo deben estar sujetos a una validación independiente

- Adecuado: facilitar la medición adecuada del desempeño y personas involucradas dentro de las diferentes áreas de la organización.

\subsection{Consecuencias del manejo inadecuado del gobierno corporativo}

Las organizaciones que manejan inadecuadamente el gobierno corporativo, generalmente poseen una tendencia a caer en trampas relacionadas con el manejo de los dueños o inversores de la empresa, mismas que estanca el funcionamiento adecuado, expansión y reduce la capacidad de fusión empresarial (Gómez, 2018).

Dentro de las principales trampas o consecuencias de un inadecuado manejo del gobierno corporativo tenemos: la sensación de no ser dueño de la empresa, pese a encontrarse o figurar como accionista, confundir el rol de propietario con administrador o gerente empresarial, tomar decisiones en base a emociones sin análisis o consulta a las áreas que conforman la organización, exceso de confianza o desconocimiento de los riesgos a los que se encuentra inmersa la organización por el cambio constante de su entorno, no realizar el manejo adecuado de la utilidad empresarial (dividendos) por no tener estándares claros de trabajo, conflicto de interés relacionados con los puestos en la organización o miedos generados por el manejo inadecuado y atrapar o sofocar a los accionistas actuales o potenciales creando malas expectativas en relación al valor de la organización (Gómez, 2018).

\subsection{Gobierno corporativo enfocado a Latinoamérica}

Las empresas latinoamericanas, en su gran mayoría, enfocan pequeñas y medianas organizaciones generalmente llamadas pymes, mismas que por su naturaleza necesitan tener un adecuado gobierno corporativo para poder crecer, desarrollarse y ganar competitividad ente posibles negociaciones y cotizaciones en las diferentes Bolsas de valores (Gómez, 2018).

El autor Gonzalo Gómez en su obra "Los retos de un buen gobierno corporativo", plantea un modelo enfocado a las PYMES latinoamericanas, enfocándose a las empresas familiares y no familiares que representan una fuerza productiva poco desarrollado administrativamente y con riesgo a caer y no ser competitivas a lo largo del tiempo.

En la Figura 3 se muestra la estructura de gobierno corporativo enfocado a las PYMES familiares de Latinoamérica. 


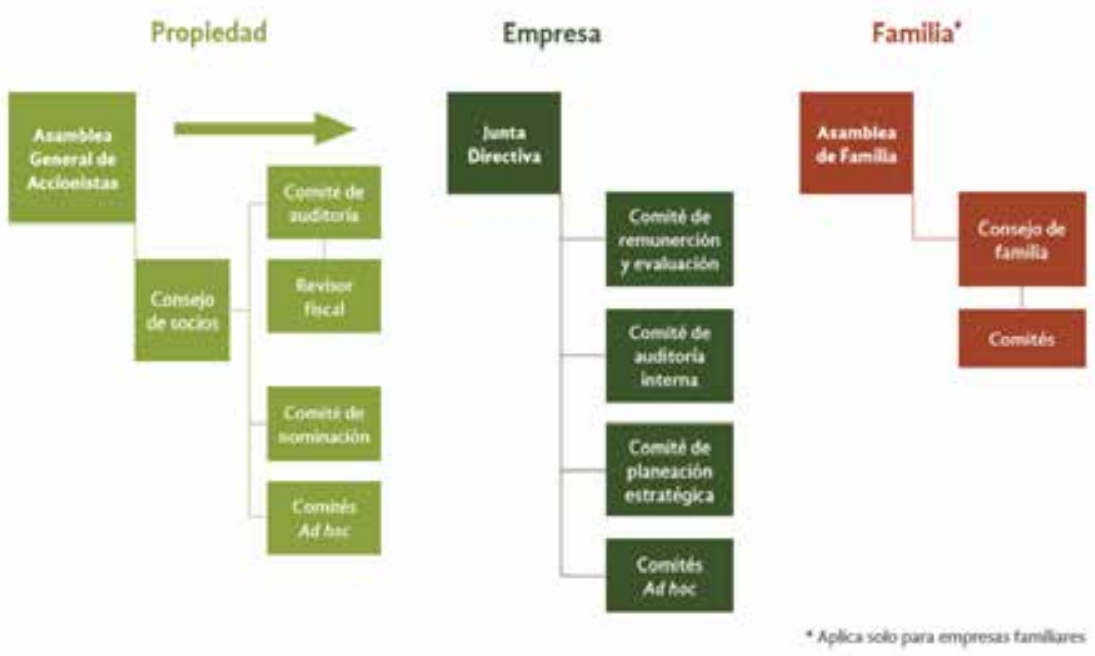

Figura 3. Estructura de gobierno corporativo enfocado a las PYMES familiares de Latinoamérica Fuente: Los retos de un buen gobierno corporativo (Gómez, 2018).

\section{Metodología}

La investigación se fundamentó en un modelo de correlación de datos mediante el análisis de antecedentes cualitativos y cuantitativos, conjuntamente se realizó un estudio descriptivo analizando las tendencias de los gobiernos corporativos de las grandes empresas ecuatorianas y su influencia en la competitividad internacional a través de los instrumentos de recolección de datos como encuestas y entrevistas a las 100 mejores empresas ecuatorianas según ranking empresariales.

Se aplicará además un tipo de investigación documental para el análisis de información proveniente de: artículos científicos, libros relacionados al tema, leyes y normas del gobierno corporativo, informes de la Superintendencia de Compañías, Superintendencia de Bancos, metodologías de la internacionalización y repositorios de trabajos de investigación realizados sobre el tema.

\section{Resultados}

\section{1. Ámbito empresarial en el Ecuador}

En el Ecuador, las organizaciones registradas legalmente hasta el año 2018, según cifras aportadas por el Instituto Nacional de Estadística y Censo (INEC), son 88.828 empresas legalmente registradas, mismas que se visualizan detalladamente en la Tabla 3. 
Tabla 3.

Empresas Registradas en el Ecuador 2019

\begin{tabular}{|l|l|l|}
\hline \multicolumn{2}{|l|}{ Empresas legalmente registradas en el Ecuador 2019} \\
\hline Tamaño de Empresa & $\begin{array}{l}\text { No. } \\
\text { Empresa }\end{array}$ & Porcentaje Empresarial \\
\hline Pequeña empresa & 61.759 & $7,00 \%$ \\
\hline Microempresa & 802.353 & $90,89 \%$ \\
\hline Mediana Empresa "A" & 8.544 & $0,97 \%$ \\
\hline Mediana Empresa "B" & 5.798 & $0,66 \%$ \\
\hline Grande Empresa & 4.312 & $0,49 \%$ \\
\hline Total & 882.766 & $100 \%$ \\
\hline
\end{tabular}

Fuente: Instituto Nacional Estadística y Censos (INEC, 2019)

Es importante resaltar que en el Ecuador el $4 \%$ de las empresas grandes, aplican en sus estructuras un sistema de gobierno corporativo. Esto se debe a que las empresas ecuatorianas en su gran mayoría son familiares, generando diversas dificultades al momento de realizar una estructura organizacional clara.

Tabla 4.

Sectores Económicos Ecuador 2018

\begin{tabular}{|l|l|l|}
\hline \multicolumn{3}{|l|}{ Actividad por sectores Económicos Ecuador 2018 } \\
\hline Sector Económico & No. Empresas & Porcentaje \\
\hline Servicios & 35926 & $40 \%$ \\
\hline Comercios & 32594 & $37 \%$ \\
\hline Industrias Manufactura & 7729 & $9 \%$ \\
\hline Agricultura, ganadería, silvicultura y pesca & 6442 & $7 \%$ \\
\hline Construcción & 5593 & $6 \%$ \\
\hline Explotación de Minas y Canteras & 544 & $1 \%$ \\
\hline TOTAL & $\mathbf{8 8 8 2 8}$ & $\mathbf{1 0 0} \%$ \\
\hline
\end{tabular}

Fuente: Instituto Nacional Estadística y Censos (INEC, 2019)

En referencia a la Tabla 4, se evidencia que en el Ecuador, el $77 \%$ de las empresas se dedican a realizar actividades relacionadas con servicios o comercialización de bienes, dejando un horizonte de posibilidades para las empresas de manufactura, agricultura, construcción y minería, mismas que al implementar el gobierno corporativo, obtendrán ventaja competitiva en los mercados nacionales e internacionales, debido a que sus procesos serán más claros, sus funciones eficaces y contarán con inversores internos o externos.

Por lo expresado anterior mente el gobierno corporativo representa una visión clara de las empresas ecuatorianas y como estas pueden obtener una ventaja competitiva a nivel mundial, debido a que el gobierno corporativo brinda herramientas para captar inversiones nacionales e internacionales, apoya a la mejora continua en los flujos de información y procesos establecidos, 
convierte a las empresas familiares en organizaciones claras y correctamente estructuradas (CMF, 2020).

Tabla 5.

Ranking de las diez mejores Empresas del Ecuador

\begin{tabular}{|c|c|c|c|c|c|}
\hline Ranking & Empresa & Ingresos totales & Utilidad bruta & $\begin{array}{l}\text { Impuesto } \\
\text { causado }\end{array}$ & $\begin{array}{l}\text { Utilidad } \\
\text { ingresos }\end{array}$ \\
\hline 1 & Corporación Favorita C.A. & $\$ 2.089 .984 .173$ & $\$ 237.195 .939$ & $\$ 49.037 .870$ & $11 \%$ \\
\hline 2 & Claro & $\$ 1.327 .121 .354$ & $\$ 234.606 .575$ & $\$ 60.744 .023$ & $18 \%$ \\
\hline 3 & Banco Pichincha & $\$ 1.244 .818 .889$ & $\$ 204.906 .980$ & $\$ 53.727 .535$ & $16 \%$ \\
\hline 4 & Corporación el Rosado S.A. & $\$ 1.154 .630 .447$ & $\$ 40.067 .111$ & $\begin{array}{l} \\
9.056 .421\end{array}$ & $3 \%$ \\
\hline 5 & Pronaca & \begin{tabular}{|l|} 
\\
967.206 .669
\end{tabular} & $\$ 74.862 .989$ & $\$ 18.524 .184$ & $8 \%$ \\
\hline 6 & $\begin{array}{l}\text { General Motors del Ecuador } \\
\text { S.A. }\end{array}$ & $\$ 926.009 .140$ & $\$ 61.929 .170$ & $\$ 13.179 .248$ & $7 \%$ \\
\hline 7 & Dinadec S.A. & $\begin{array}{l}\$ \\
911.506 .769\end{array}$ & \$ 32.499 .961 & $\begin{array}{l}\$ \\
7.362 .935\end{array}$ & $4 \%$ \\
\hline 8 & $\begin{array}{l}\text { Distribuidora farmacéutica } \\
\text { ecuatoriana (DIFARE) S.A. }\end{array}$ & $\begin{array}{l}\$ \\
727.119 .205\end{array}$ & $\$ 26.726 .079$ & $\begin{array}{l}\$ \\
6.364 .249\end{array}$ & $4 \%$ \\
\hline 9 & Tía & $\$ \quad 687.780 .606$ & $\$ 64.275 .423$ & $\$ 18.281 .207$ & $9 \%$ \\
\hline 10 & Telefónica Movistar & $\begin{array}{l}\text { \$ } \\
652.231 .887\end{array}$ & $\$ \quad 2.400 .000$ & $\$ 22.438 .098$ & $0 \%$ \\
\hline
\end{tabular}

Fuente: Superintendencia de Compañías, Valores y Seguros (2019)

Un estudio realizado por la superintendencia de compañías en el año 2018, determino el ranking de las mejores empresas del Ecuador (Tabla 5), mismas que cuentan con un modelo claro de gobierno corporativo, facilitando su expansión y presencia a nivel nacional e internacional, esto se visualiza en cada una de sus páginas web, dando confianza a los inversores de contar con un modelo de negocios claro y bien definido (ECURED, 2020).

\subsection{Elementos del gobierno corporativo ecuatoriano}

El gobierno corporativo comprende un conjunto de normas y prácticas referente a la estructura y procesos para la dirección de las compañías, el contar dentro de las organizaciones con un consejo de administración asegura la rendición de cuentas, equidad y transparencia en todas las partes interesadas. Los elementos de un gobierno corporativo son la transparencia, la visión estratégica, responsabilidad, igualdad de los accionistas y la responsabilidad social (Magallanes, 2017, pág. 1).

Este consiste en la unión de acuerdos, factores de poder y políticas creadas ante situaciones cambiantes que se pueden presentar en el ambiente empresarial, conjuntamente coordina los responsables de las áreas gerenciales y sus funciones específicas. Está estrechamente asociado 
con las operaciones matutinas que realizan las instituciones públicas y privadas sin importar su tamaño o actividad económica (Urriza, 2017, pág. 12).

En el Ecuador todavía no está muy considerado el gobierno corporativo dentro de las organizaciones, muchas de ellas no han podido expandirse a nivel internacional y han perdido competitividad ante un mercado globalizado y simplemente han sido absorbidas, compradas, o no tienen más crecimiento que el mercado nacional (Culqui, 2013).

Tal como sucede con muchos de los temas relacionados con la globalización, las preocupaciones y las esperanzas sobre la inversión internacional giran en torno, de diferentes formas, a lo que los gobiernos puedan hacer. Ello significa la implementación de un buen gobierno corporativo con directivos idóneos que ayuden a fortalecer las organizaciones.

\subsection{Análisis de correlación y relación entre gobierno corporativo y competitividad empresarial}

En la presente investigación se aplicó el análisis coeficiente de correlación Tau de Kendall mismo que aportara de manera clara la relación existente entre el gobierno corporativo de las empresas ecuatorianas en sus diferentes niveles y el impacto en la competitividad empresarial, datos que se plasman en la Tabla 6.

Tabla 6.

Sectores Económicos Ecuador 2019

\begin{tabular}{|l|r|r|r|r|r|}
\hline Tamaño de Empresa & No. Empresas & $\begin{array}{c}\text { Porcentaje } \\
\text { Empresar- } \\
\text { ial }\end{array}$ & $\begin{array}{c}\text { Utilidades Mil- } \\
\text { lones }\end{array}$ & $\begin{array}{c}\text { Porcentaje de } \\
\text { Utilidades }\end{array}$ & $\begin{array}{c}\text { Aplicación del } \\
\text { Gobierno Cor- } \\
\text { porativo }\end{array}$ \\
\hline Pequeña empresa & 61.759 & $7,00 \%$ & $\$ 18.558,00$ & $11 \%$ & $0 \%$ \\
\hline Microempresa & 802.353 & $90,89 \%$ & $\$ 1.534,00$ & $1 \%$ & $0 \%$ \\
\hline Mediana Empresa “A” & 8.544 & $0,97 \%$ & $\$ 10.376,00$ & $10 \%$ & $60 \%$ \\
\hline Mediana Empresa "B" & 5.798 & $0,66 \%$ & $\$ 16.454,00$ & $72 \%$ & $90 \%$ \\
\hline Grandes Empresa & 4.312 & $0,49 \%$ & $\$ 122.529,00$ & $\mathbf{1 0 0} \%$ & $100 \%$ \\
\hline \multicolumn{1}{|c|}{ Total } & $\mathbf{8 8 2 . 7 6 6}$ & $\mathbf{1 0 0} \%$ & $\mathbf{\$ 1 6 9 . 4 5 1 , 0 0}$ & & \\
\hline
\end{tabular}

Fuente: Instituto Nacional Estadística y Censos (INEC, 2019) / Revista Líderes (Gobierno Corporativo en la comunidad empresarial) 

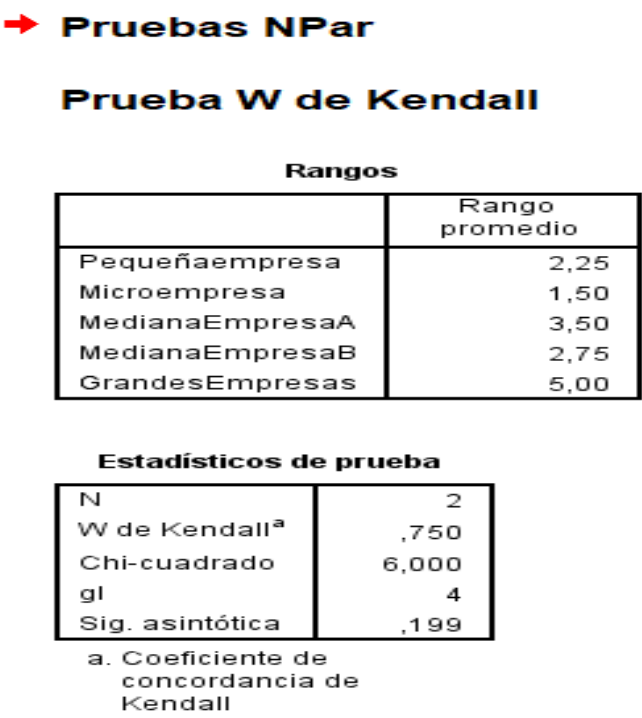

Figura 4. Resultados del Análisis de Correlación W de Kendall SPSS

Fuente: Analisis del autor y resultados obtenidos por el SPSS

En base a la Figura 4, se puede determinar que con un valor de 0.750 de análisis de correlación Kendal el gobierno corporativo y las utilidades empresariales están directamente relacionadas, de este modo si el gobierno corporativo se aplica en las empresas pequeñas y microempresas, sus utilidades se verían incrementadas considerablemente, determinando la influencia del mismo en la competitividad nacional e internacional de las organizaciones ecuatorianas.

\subsection{Prueba y verificación de hipótesis}

En base a los resultados obtenidos en el análisis de correlación Kendall se procedió a la verificación y prueba de hipótesis mediante la herramienta estadística JI Cuadrada, misma que nos aportara una prueba y verificación exacta de la hipótesis planteada en el presente artículo (Fernández Sampieri \& Mendoza Torres, 2018).

$$
\begin{gathered}
X^{2}=K(N-1) W \\
X_{0.05}^{2}=2(5-1) * 0.750 \\
X_{0.05}^{2}=6.00
\end{gathered}
$$

Figura 5. Formula y Aplicación del análisis estadístico Jl Cuadrada

Fuente: Analisis del Autor y resultados obtenidos por el SPSS

En base a los valores en tablas estadísticos con grado de confianza del 95\% se determinó que el gobierno corporativo influye en la competitividad empresarial, aportando un incremento considerable en sus utilidades y manejo de la capacidad administrativa. 


\section{Conclusiones}

El gobierno corporativo influye con un porcentaje del 95\% en la competitividad de las empresas ecuatorianas, de ese modo las organizaciones que mantienen una correcta aplicación tienden a desarrollarse y mejorar sus posibilidades de internacionalización.

La competitividad nacional e internacional se encuentra directamente relacionada con el gobierno corporativo, de este modo es indispensable su implementación total en las pequeñas y medianas empresas bajo un modelo de asociatividad que les permita agruparse, enfrentar y sobrellevar los retos actuales que aporta la globalización.

El gobierno corporativo permite a las organizaciones, contar con una ventaja competitiva ante la globalización y la necesidad que las mismas pueden tener para optimizar sus sistemas y procesos a lo largo de sus actividades.

El gobierno corporativo representa un modelo de transparencia para los inversionistas, por lo cual las empresas se vuelven atractivas en su gestión de desarrollo empresarial.

En el Ecuador el $4 \%$ de las empresas pertenecen a las grandes corporaciones, mismas que al contar con un sistema claro de gobierno corporativo tienen mejores garantías de inversión, mayores oportunidades de expiación y una mejora continua en cada uno de sus procesos, logrando un posicionamiento a nivel nacional e internacional.

Los gobiernos locales, cuentan con diversos lineamientos para que las empresas incorporen el gobierno corporativo, pero muchas veces por factores culturales o socioeconómicos no son considerados, esto se refleja en las pequeñas y medianas empresas, mismas que se crean muchas veces de forma familiar, dificultando el estructurar de manera clara el orden jerárquico, que es fundamental en la aplicación de este sistema.

El gobierno corporativo, brinda a las empresas una frontera de posibilidades mismas que se ven reflejadas en las posibilidades de captar nuevos capitales económicos, optimizar sus estándares y producción, generar una mejora continua y obtener una ventaja competitiva en mercados globalizados.

Por lo expresado anteriormente el gobierno corporativo ha aplicado estrategias de asociatividad empresarial que impulsaría a las pequeñas y medianas empresas a desarrollarse y mejorar en sus procesos administrativos, ventas y comercialización, generando la expansión progresiva de las mismas en el mercado nacional e internacional.

La corrupción empresarial de los sectores públicos y privados ha llevado que la administración de los modelos empresariales sean pocos consistentes o se vuelvan desconfiables, cada día los empresarios buscan en tener herramientas y procesos que eviten la corrupción en toda su cadena de valor. Con una estructura de gobierno de corporativo basado en una filosofía, ética, responsabilidad y valores lograremos la transparencia de su administración y consigo su competitividad. 
Tomar como referencia los modelos de gobierno corporativo de otros países, nos podría proporcionar algunas buenas prácticas, que se crearía un modelo propio para la empresa ecuatoriana acorde las exigencias de los inversionistas. 


\section{Referencias}

Acción y Desarrollo. (2020). DEFINICION DE BANCO COMUNAL. 1. Obtenido de http://www.accionydesarrollo. fin.ec/index.php/servicios/credito/para-cajas-comunitarias

Bernal, M. C. (2019). Las tecnologías de la información y comunicación como factor de innovación y competitividad empresarial. Universidad Técnologica de Pereira, 11. Obtenido de http://revistas.utp.edu.co/index. php/revistaciencia/article/view/20401/14281

Bolsa de Comercio de Buenos Aires. (2020). Fideicomisos financieros. Bolsa de Comercio de Buenos Aires, 1. Obtenido de https://www.bcba.sba.com.ar/capacitacion/invertir/en-que-invertir/fideicomisos-financieros/

Cárdenas, G. (2018). Buenas prácticas en la gestión del gobierno corporativo. Conexionesan, pág. 1.

Caso aplicado para empresas del Sector Norte Industrial del Distrito Metropolitano de Quito, 1 (1 2013).

CMF. (2020). ¿Qué es un Banco? Portal de Edicación Financiera, 1. Obtenido de https://www.cmfeduca.cl/educa/600/w3-article-27422.html

Culqui, G. (2013). Las cooperativas de ahorro y crédito y su incidencia sobre el ahorro del Ecuador. Escuela Politécnica Nacional, 13. Obtenido de https://bibdigital.epn.edu.ec/bitstream/15000/7086/1/CD-5264.pdf

ECURED. (2020). Instituciones Financieras. ECURED, 1. Obtenido de https://www.ecured.cu/Instituciones_financieras

Expansión. (2019). Indice de Competitivida Global. Obtenido de Expansió/datos macro.com: https://datosmacro. expansion.com/estado/indice-competitividad-global/ecuador\#: :text=Ecuador\%20baja\%20al\%20puesto\%2090\%C2\%BA,un\%20alto\%20nivel\%20de\%20prosperidad.

Fernández Sampieri, R., \& Mendoza Torres, C. (2018). Metodología de la Investigación: las rutas cuantitativa, cualitativa y mixta. Mexico: McGraw Hill.

Gómez, G. (2018). Los retos de un buen gobierno corporativo. lymgroup, 3.

INEC. (2019). Directorio de Empresas. Instituto Nacional Estadística y Censos. Obtenido de https://www.ecuadorencifras.gob.ec/directoriodeempresas/

López, S. P. (2020). Regulación en contabilidad y gobierno corporativo en la Znanciarización de empresas. Cuadernos de Contabilidad, 20. Obtenido de https://dialnet.unirioja.es/servlet/articulo?codigo=7458715

Magallanes, V. (2017). Los Cinco Elementos Del Gobierno Corporativo. Gestión Solidaria, 1.

OCDE. (2016). Principios de Gobierno de la OCDE y del G20. Paris: Éditions OCDE. 
Peña, S. (26 de enero de 2020). Influencia del Gobierno Corporativo en las Empresas Familiares en su comportamiento contable -financiero, en crisis económicas y lo sanitarias: Ecuador, periodo 2020. EspírituEmprendedor, 24. Obtenido de http://206.189.217.80/index.php/revista/article/view/223/266

Peralta, P. (2020). En Ecuador, la inversión extranjera directa sigue estancada. Pichincha Comunicaciones, 1.

Porter, M. (1990). The competitive advantage of nations. ISBN: 0-684-8446-0, 2.

Sisa, F. G. (15 de Junio de 2020). Asociatividad empresarial: estrategia para la competitividad de las PYMES en el Ecuador. ERUDITUS, 1-12. Obtenido de https://revista.uisrael.edu.ec/index.php/re/article/view/308/162

Superintendencia de Compañías, Valores y Seguros. (2019). RANKING EMPRESARIAL 2018. Recuperado el 16 de diciembre de 2019, de https://appscvs.supercias.gob.ec/rankingCias/principal.zul

UNCTAD, C. d. (2004). Transparencia. Ginebra: Naciones Unidas.

Urriza, M. N. (Septiembre de 2017). Gobernabilidad y Gobierno Corporativo. Revista Adenag, 12. Obtenido de http://200.49.237.216/bitstream/123456789/4560/3/Gobernabilidad\%20y\%20gobierno\%20corporativo\%20an\%c3\%a1lisis\%20y\%20medici\%c3\%b3n\%20de\%20su\%20eficacia\%20en\%20organizaciones. pdf

Urriza, M. N. (Septiembre de 2017). Gobernabilidad y Gobierno Corporativo. Revista Adenag, 12. Obtenido de http://200.49.237.216/bitstream/123456789/4560/3/Gobernabilidad\%20y\%20gobierno\%20corporativo\%20an\%c3\%a1lisis\%20y\%20medici\%c3\%b3n\%20de\%20su\%20eficacia\%20en\%20organizaciones. pdf 